PROCEEDINGS OF THE

AMERICAN MATHEMATICAL SOCIETY

Volume 139, Number 10, October 2011, Pages 3657-3664

S 0002-9939(2011)10913-4

Article electronically published on March 9, 2011

\title{
REDUCIBILITY OF THE WAVELET REPRESENTATION ASSOCIATED TO THE CANTOR SET
}

\author{
DORIN ERVIN DUTKAY AND SERGEI SILVESTROV
}

(Communicated by Richard Rochberg)

\begin{abstract}
We answer a question by Judith Packer about the irreducibility of the wavelet representation associated to the Cantor set. We prove that if the QMF filter does not have constant absolute value, then the wavelet representation is reducible.
\end{abstract}

\section{INTRODUCTION}

Wavelet representations were introduced in Jor01, Dut02, DJ07b in an attempt to apply the multiresolution techniques of wavelet theory Dau92 to a larger class of problems where self-similarity, or refinement is the central phenomenon. They were used to construct wavelet bases and multiresolutions on fractal measures and Cantor sets [DJ06] or on solenoids [Dut06].

Wavelet representations can be defined axiomatically as follows: let $X$ be a compact metric space and let $r: X \rightarrow X$ be a Borel measurable function which is onto and finite-to-one; i.e., $0<\# r^{-1}(x)<\infty$ for all $x \in X$. Let $\mu$ be a strongly invariant measure on $X$; i.e.,

$$
\int_{X} f d \mu=\int_{X} \frac{1}{\# r^{-1}(x)} \sum_{r(y)=x} f(y) d \mu(x), \quad\left(f \in L^{\infty}(X)\right) .
$$

Let $m_{0} \in L^{\infty}(X)$ be a $Q M F$ filter; i.e.,

$$
\frac{1}{\# r^{-1}(x)} \sum_{r(y)=x}\left|m_{0}(y)\right|^{2}=1 \text { for } \mu \text {-a.e. } x \in X \text {. }
$$

Theorem 1 (DJ07b] $)$. There exists a Hilbert space $\mathcal{H}$, a unitary operator $U$ on $\mathcal{H}$, a representation $\pi$ of $L^{\infty}(X)$ on $\mathcal{H}$ and an element $\varphi$ of $\mathcal{H}$ such that

(1) (Covariance) $U \pi(f) U^{-1}=\pi(f \circ r)$ for all $f \in L^{\infty}(X)$.

(2) (Scaling equation) $U \varphi=\pi\left(m_{0}\right) \varphi$.

(3) (Orthogonality) $\langle\pi(f) \varphi, \varphi\rangle=\int f d \mu$ for all $f \in L^{\infty}(X)$.

(4) (Density) $\left\{U^{-n} \pi(f) \varphi \mid n \in \mathbb{N}, f \in L^{\infty}(X)\right\}$ is dense in $\mathcal{H}$.

Moreover they are unique up to isomorphism.

Received by the editors September 4, 2010.

2010 Mathematics Subject Classification. Primary 42C40, 28D05, 47A67, 28A80.

Key words and phrases. Wavelet representation, quadrature mirror filter, Cantor set.

Research supported in part by the Swedish Foundation for International Cooperation in Research and Higher Education (STINT), the Swedish Research Council 621-2007-6338, the Swedish Royal Academy of Sciences and the Crafoord Foundation.

(C)2011 American Mathematical Society Reverts to public domain 28 years from publication 
Definition 2. We say that $(\mathcal{H}, U, \pi, \varphi)$ in Theorem 1 is the wavelet representation associated to $m_{0}$.

Our main focus will be the irreducibility of the wavelet representation.

The most familiar wavelet representation is the classical one on $L^{2}(\mathbb{R})$, where $U$ is the operator of dilation by 2 and $\pi$ is obtained by applying the Borel functional calculus to the translation operator $T$; i.e., $\pi(f)=f(T)$ for $f$ a bounded function on $\mathbb{T}$, the unit circle. This representation is associated to the map $r(z)=z^{2}$ on $\mathbb{T}$, the measure $\mu$ is just the Haar measure on the circle, and $m_{0}$ can be any lowpass QMF filter which produces an orthogonal scaling function (see [Dau92]). For example, one can take the Haar filter $m_{0}(z)=(1+z) / \sqrt{2}$ which produces the Haar scaling function $\varphi$.

This representation is reducible; its commutant was computed in [HL00] and the direct integral decomposition was presented in LPT01.

Some low-pass filters, such as the stretched Haar filter $m_{0}(z)=\left(1+z^{3}\right) / \sqrt{2}$, give rise to non-orthogonal scaling functions. In this case super-wavelets appear, and the wavelet representation is realized on a direct sum of finitely many copies of $L^{2}(\mathbb{R})$. See BDP05. This representation is also reducible and its direct integral decomposition is similar to the one for $L^{2}(\mathbb{R})$. See BDP05, Dut06].

When one takes the QMF filter $m_{0}=1$ the situation is very different. As shown in Dut06, the representation can be realized on a solenoid, and in this case it is irreducible. The result holds even for more general maps $r$ if they are ergodic (see [DLS09]).

The general theory of the decomposition of wavelet representations into irreducible components was given in Dut06, but there is a large class of examples where it is not known whether these representations are irreducible or not.

One interesting example, introduced in [DJ07a], is the following: take the map $r(z)=z^{3}$ on the unit circle $\mathbb{T}$ with the Haar measure $\mu$. Consider the QMF filter $m_{0}(z)=\left(1+z^{2}\right) / \sqrt{2}$. The wavelet representation associated to this data is strongly connected to the middle-third Cantor set. It can be realized as follows:

Let $\mathbf{C}$ be the middle-third Cantor set. Let

$$
\mathcal{R}:=\bigcup\left\{\mathbf{C}+\frac{k}{3^{n}} \mid k, n \in \mathbb{Z}\right\} .
$$

Let $\mathcal{H}^{s}$ be the Hausdorff measure of dimension $s:=\log _{3} 2$, i.e., the Hausdorff dimension of the Cantor set. Restrict $\mathcal{H}^{s}$ to the set $\mathcal{R}$. Consider the Hilbert space $\mathcal{H}:=L^{2}\left(\mathcal{R}, \mathcal{H}^{s}\right)$. Define the unitary operators on $\mathcal{H}$,

$$
U f(x)=\frac{1}{\sqrt{2}} f\left(\frac{x}{3}\right), \quad T f(x)=f(x-1),
$$

and define the representation $\pi$ of $L^{\infty}(\mathbb{T})$ on $\mathcal{H}$ by applying Borel functional calculus to the operator $T: \pi(f)=f(T)$ for $f \in L^{\infty}(X)$.

The scaling function is defined as the characteristic function of the Cantor set $\varphi:=\chi_{\mathbf{C}}$.

Then $(\mathcal{H}, U, \pi, \varphi)$ is the wavelet representation associated to the QMF filter $m_{0}(z)=\left(1+z^{2}\right) / \sqrt{2}$.

In February 2009, at the FL-IA-CO-OK workshop in Iowa City, following investigations into general multiresolution theories BFMP09b, BFMP09a, BLP09, BLM09, Judith Packer formulated the following question: is this representation irreducible? We will answer this question here and show that the representation 
is not irreducible. Indeed, we show that $m_{0}=1$ is an exception and, under some mild assumptions, all the other QMF filters give rise to reducible representations.

In [DLS09], several equivalent forms of this problem were presented in terms of refinement equations, fixed points of transfer operators or ergodic shifts on solenoids. Using the results in DLS09 we obtain as a corollary non-trivial solutions to all these problems.

\section{MAIN RESULT}

Theorem 3. Suppose $r:(X, \mu) \rightarrow(X, \mu)$ is ergodic. Assume $\left|m_{0}\right|$ is not constant equal to $1 \mu$-a.e., non-singular, i.e., $\mu\left(m_{0}(x)=0\right)=0$, and $\log \left|m_{0}\right|^{2}$ is in $L^{1}(X)$. Then the wavelet representation $(\mathcal{H}, U, \pi, \varphi)$ is reducible.

Proof. We recall some facts from DJ07b]. The wavelet representation can be realized on a solenoid as follows: Let

$$
X_{\infty}:=\left\{\left(x_{0}, x_{1}, \ldots\right) \in X^{\mathbb{N}} \mid r\left(x_{n+1}\right)=x_{n} \text { for all } n \geq 0\right\} .
$$

We call $X_{\infty}$ the solenoid associated to the map $r$.

On $X_{\infty}$ consider the $\sigma$-algebra generated by cylinder sets. Define the map $r_{\infty}$ : $X_{\infty} \rightarrow X_{\infty}$ as follows:

$$
r_{\infty}\left(x_{0}, x_{1}, \ldots\right)=\left(r\left(x_{0}\right), x_{0}, x_{1}, \ldots\right) \text { for all }\left(x_{0}, x_{1}, \ldots\right) \in X_{\infty} .
$$

Then $r_{\infty}$ is a measurable automorphism on $X_{\infty}$.

Define $\theta_{0}: X_{\infty} \rightarrow X$,

$$
\theta_{0}\left(x_{0}, x_{1}, \ldots\right)=x_{0} .
$$

The measure $\mu_{\infty}$ on $X_{\infty}$ will be defined by constructing some path measures $P_{x}$ on the fibers $\Omega_{x}:=\left\{\left(x_{0}, x_{1}, \ldots\right) \in X_{\infty} \mid x_{0}=x\right\}$.

Let

$$
c(x):=\# r^{-1}(r(x)), \quad W(x)=\left|m_{0}(x)\right|^{2} / c(x), \quad(x \in X) .
$$

Then

$$
\sum_{r(y)=x} W(y)=1, \quad(x \in X) .
$$

$W(y)$ can be thought of as the transition probability from $x=r(y)$ to one of its roots $y$.

For $x \in X$, the path measure $P_{x}$ on $\Omega_{x}$ is defined on cylinder sets by

$$
P_{x}\left(\left\{\left(x_{n}\right)_{n \geq 0} \in \Omega_{x} \mid x_{1}=z_{1}, \ldots, x_{n}=z_{n}\right\}\right)=W\left(z_{1}\right) \ldots W\left(z_{n}\right)
$$

for any $z_{1}, \ldots, z_{n} \in X$.

This value can be interpreted as the probability of the random walk going from $x$ to $z_{n}$ through the points $x_{1}, \ldots, x_{n}$.

Next, define the measure $\mu_{\infty}$ on $X_{\infty}$ by

$$
\int f d \mu_{\infty}=\int_{X} \int_{\Omega_{x}} f\left(x, x_{1}, \ldots\right) d P_{x}\left(x, x_{1}, \ldots\right) d \mu(x)
$$

for bounded measurable functions on $X_{\infty}$.

Consider now the Hilbert space $\mathcal{H}:=L^{2}\left(\mu_{\infty}\right)$. Define the operator

$$
U \xi=\left(m_{0} \circ \theta_{0}\right) \xi \circ r_{\infty}, \quad\left(\xi \in L^{2}\left(X_{\infty}, \mu_{\infty}\right)\right) .
$$


Define the representation of $L^{\infty}(X)$ on $\mathcal{H}$ :

$$
\pi(f) \xi=\left(f \circ \theta_{0}\right) \xi, \quad\left(f \in L^{\infty}(X), \xi \in L^{2}\left(X_{\infty}, \mu_{\infty}\right)\right) .
$$

Let $\varphi=1$ be the constant function 1 .

Lemma 4 (DJ07b] $)$. Suppose $m_{0}$ is non-singular, i.e.,

$$
\mu\left(\left\{x \in X \mid m_{0}(x)=0\right\}\right)=0 .
$$

Then the data $(\mathcal{H}, U, \pi, \varphi)$ forms the wavelet representation associated to $m_{0}$.

We proceed to the proof of our main result.

From the QMF relation and the strong invariance of $\mu$ we have

$$
\int_{X}\left|m_{0}\right|^{2} d \mu=\int_{X} \frac{1}{\# r^{-1}(x)} \sum_{r(y)=x}\left|m_{0}(y)\right|^{2} d \mu=1
$$

By Jensen's inequality we have

$$
a:=\int_{X} \log \left|m_{0}\right|^{2} d \mu \leq \log \int_{X}\left|m_{0}\right|^{2} d \mu=0 .
$$

Since $\log$ is strictly concave and $\left|m_{0}\right|^{2}$ is not constant $\mu$-a.e., it follows that the inequality is strict and $a<0$.

Since $r$ is ergodic, applying Birkoff's ergodic theorem, we obtain that

$$
\lim _{n \rightarrow \infty} \frac{1}{n} \sum_{k=0}^{n-1} \log \left|m_{0} \circ r^{k}\right|^{2}=\int_{X} \log \left|m_{0}\right|^{2} d \mu=a, \mu-\text { a.e. }
$$

This implies that

$$
\lim _{n \rightarrow \infty}\left(\left|m_{0}(x) m_{0}(r(x)) \ldots m_{0}\left(r^{n-1}(x)\right)\right|^{2}\right)^{1 / n}=e^{a}<1, \mu-\text { a.e. }
$$

Take $b$ with $e^{a}<b<1$.

By Egorov's theorem, there exists a measurable set $A_{0}$ such that $\mu_{\infty}\left(A_{0}\right)>0$ and $\left(\left|m_{0}(x) m_{0}(r(x)) \ldots m_{0}\left(r^{n-1}(x)\right)\right|^{2}\right)^{1 / n}$ converges uniformly to $e^{a}$ on $A_{0}$. This implies that there exists an $n_{0}$ such for all $m \geq n_{0}$ :

$$
\left(\left|m_{0}(x) m_{0}(r(x)) \ldots m_{0}\left(r^{m-1}(x)\right)\right|^{2}\right)^{1 / m} \leq b \text { for } x \in A_{0}
$$

and so

$$
\begin{array}{r}
\left|m_{0}(x) m_{0}(r(x)) \ldots m_{0}\left(r^{m-1}(x)\right)\right|^{2} \leq b^{m}, \\
\text { for } m \geq n_{0} \text { and all } x \in A_{0} .
\end{array}
$$

Next, given $m \in \mathbb{N}$, we compute the probability of a sequence $\left(z_{n}\right)_{n \in \mathbb{N}} \in X_{\infty}$ having $z_{m} \in A_{0}$. We have, using the strong invariance of $\mu$ :

$$
\begin{gathered}
P\left(z_{m} \in A_{0}\right)=\mu_{\infty}\left(\left\{\left(z_{n}\right)_{n} \mid z_{m} \in A_{0}\right\}\right)=\int_{X_{\infty}} \chi_{A_{0}} \circ \theta_{m} d \mu_{\infty} \\
=\int_{X} \frac{1}{\# r^{-m}\left(z_{0}\right)} \sum_{r\left(z_{1}\right)=z_{0}, \ldots, r\left(z_{m}\right)=z_{m-1}}\left|m_{0}\left(z_{1}\right)\right|^{2} \ldots\left|m_{0}\left(z_{m}\right)\right|^{2} \chi_{A_{0}}\left(z_{m}\right) d \mu\left(z_{0}\right) \\
=\int_{X}\left|m_{0}\left(z_{m}\right) m_{0}\left(r\left(z_{m}\right)\right) \ldots m_{0}\left(r^{m-1}\left(z_{m}\right)\right)\right|^{2} \chi_{A_{0}}\left(z_{m}\right) d \mu\left(z_{m}\right) \\
=\int_{X}\left|m_{0}(x) m_{0}(r(x)) \ldots m_{0}\left(r^{m-1}(x)\right)\right|^{2} \chi_{A_{0}}(x) d \mu(x) .
\end{gathered}
$$


Then

$$
\begin{gathered}
\sum_{m=1}^{\infty} P\left(z_{m} \in A_{0}\right) \\
=\sum_{m \geq 1} \int_{X}\left|m_{0}(x) m_{0}(r(x)) \ldots m_{0}\left(r^{m-1}(x)\right)\right|^{2} \chi_{A_{0}} d \mu(x)<\infty,
\end{gathered}
$$

and we use (11) in the last inequality.

Now we can use Borel-Cantelli's lemma to conclude that the probability that $z_{m} \in A_{0}$ infinitely often is zero. Thus, for $\mu_{\infty}$-a.e., $z:=\left(z_{n}\right)_{n}$, there exists $k_{z}$ (depending on the point) such that $z_{n} \notin A_{0}$ for $n \geq k_{z}$.

Suppose now that the representation is irreducible. Then $r_{\infty}$ is ergodic on $\left(X_{\infty}, \mu_{\infty}\right)$. So $r_{\infty}^{-1}$ is too. Using Birkhoff's ergodic theorem, it follows that, $\mu_{\infty}$-a.e.,

$$
\lim _{n \rightarrow \infty} \frac{1}{n} \sum_{k=0}^{n-1}\left(\chi_{A_{0}} \circ \theta_{0}\right) \circ r_{\infty}^{-k}=\int_{X_{\infty}} \chi_{A_{0}} \circ \theta_{0} d \mu_{\infty}=\mu\left(A_{0}\right)>0 .
$$

But $\left[\left(\chi_{A_{0}} \circ \theta_{0}\right) \circ r_{\infty}^{-k}\right]\left(z_{n}\right)_{n}=\chi_{A_{0}}\left(z_{k}\right)=0$, for $k \geq k_{z}$. Therefore the sum on the left of (12) is bounded by $k_{z}$, so the limit is zero, a contradiction. Thus the representation has to be reducible.

Using the results from DLS09, we obtain that there are non-trivial solutions to refinement equations and non-trivial fixed points for transfer operators:

Corollary 5. Let $m_{0}$ be as in Theorem 3 and let $(\mathcal{H}, U, \pi, \varphi)$ be the associated wavelet representation. Then

(1) There exist solutions $\varphi^{\prime} \in \mathcal{H}$ for the scaling equation

$$
U \varphi^{\prime}=\pi\left(m_{0}\right) \varphi^{\prime}
$$

which are not constant multiples of $\varphi$.

(2) There exist non-constant, bounded fixed points for the transfer operator

$$
R_{m_{0}} f(x)=\frac{1}{\# r^{-1}(x)} \sum_{r(y)=x}\left|m_{0}(y)\right|^{2} f(y), \quad\left(f \in L^{\infty}(X), x \in X\right) .
$$

Remark 6. As shown in DJ07b], operators in the commutant of $\{U, \pi\}$ are multiplication operators $M_{g}$, with $g \in L^{\infty}\left(X_{\infty}, r_{\infty}\right)$ and $g=g \circ r_{\infty}$. Therefore, if $\mathcal{K}$ is a subspace which is invariant for $U$ and $\pi(f)$ for all $f \in L^{\infty}(X)$, then the orthogonal projection onto $\mathcal{K}$ is an operator in the commutant and so it corresponds to a multiplication by a characteristic function $\chi_{A}$, where $A$ is an invariant set for $r_{\infty}$; i.e., $A=r_{\infty}^{-1}(A)=r_{\infty}(A), \mu_{\infty}$-a.e., and $\mathcal{K}=L^{2}\left(A, \mu_{\infty}\right)$

In conclusion, the study of invariant spaces for the wavelet representation $\{U, \pi\}$ is equivalent to the study of the invariant sets for the dynamical system $r_{\infty}$ on $\left(X_{\infty}, \mu_{\infty}\right)$.

Proposition 7. Under the assumptions of Theorem 3, there are no finite dimensional invariant subspaces for the wavelet representation.

Proof. We reason by contradiction. Suppose $\mathcal{K}$ is a finite dimensional invariant subspace. Then, as in Remark 6 , this will correspond to a set $A$ invariant under $r_{\infty}, \mathcal{K}=L^{2}\left(A, \mu_{\infty}\right)$. But if $\mathcal{K}$ is finite dimensional, then $A$ must contain only atoms. Let $\left(z_{n}\right)_{n \in \mathbb{N}}$ be such an atom. We have

$$
0<\mu_{\infty}\left(\left(z_{n}\right)_{n \in \mathbb{N}}\right)=\mu\left(z_{0}\right) P_{z_{0}}\left(\left(z_{n}\right)_{n \in \mathbb{N}}\right)
$$


so $z_{0}$ is an atom for $\mu$. Since $\mu$ is strongly invariant for $\mu$, it follows that it is also invariant for $\mu$. Then $\mu\left(r\left(z_{0}\right)\right)=\mu\left(r^{-1}\left(r\left(z_{0}\right)\right)\right) \geq \mu\left(z_{0}\right)$. By induction, $\mu\left(r^{n+1}\left(z_{0}\right)\right) \geq \mu\left(r^{n}\left(z_{0}\right)\right)$. Since $\mu(X)<\infty$ and $\mu\left(z_{0}\right)>0$ this implies that for some $n \in \mathbb{N}$ and $p>0$ we have $r^{n+p}\left(z_{0}\right)=r^{n}\left(z_{0}\right)$. We relabel $r^{n}\left(z_{0}\right)$ by $z_{0}$ so we have $r^{p}\left(z_{0}\right)=z_{0}$ and $\mu\left(z_{0}\right)>0$.

Since $\mu$ is invariant for $r$ we have $\mu\left(z_{0}\right) \leq \mu\left(r^{-p}\left(z_{0}\right)\right)=\mu\left(z_{0}\right)$, and this shows that all the points in $r^{-p}\left(z_{0}\right)$ except $z_{0}$ have measure $\mu=0$. The same can be said for $r\left(z_{0}\right), \ldots, r^{p-1}\left(z_{0}\right)$. But then $C:=\left\{z_{0}, r\left(z_{0}\right), \ldots, r^{p-1}\left(z_{0}\right)\right\}$ is invariant for $r, \mu$-a.e., and has positive measure. Since $r$ is ergodic, this shows that $C=X$, $\mu$-a.e., and so we can consider that $\# r^{-1}(x)=1$ for $\mu$-a.e. $x \in X$. Then the QMF condition implies that $\left|m_{0}\right|=1 \mu$-a.e., which contradicts the assumptions in the hypothesis.

\section{EXAmples}

Example 8. Consider the map $r(z)=z^{2}$ on the unit circle $\mathbb{T}=\{z \in \mathbb{C}:|z|=1\}$. Let $\mu$ be the Haar measure on $\mathbb{T}$. Let $m_{0}(z)=\frac{1}{\sqrt{2}}(1+z)$ be the Haar low-pass filter or any filter that generates an orthonormal scaling function in $L^{2}(\mathbb{R})$ (see [Dau92]). Then the wavelet representation associated to $m_{0}$ can be realized on the Hilbert space $L^{2}(\mathbb{R})$. The dilation operator is

$$
U \xi(x)=\frac{1}{\sqrt{2}} \xi\left(\frac{x}{2}\right), \quad\left(x \in \mathbb{R}, \xi \in L^{2}(\mathbb{R})\right) .
$$

The representation $\pi$ of $L^{\infty}(\mathbb{T})$ is constructed by applying Borel functional calculus to the translation operator

$$
\begin{gathered}
T \xi(x)=\xi(x-1), \quad\left(x \in \mathbb{R}, f \in L^{2}(\mathbb{R})\right), \\
\pi(f)=f(T), \quad\left(f \in L^{\infty}(\mathbb{R})\right),
\end{gathered}
$$

in particular

$$
\pi\left(\sum_{k \in \mathbb{Z}} a_{k} z^{k}\right)=\sum_{k \in \mathbb{Z}} a_{k} T^{k}
$$

for any finitely supported sequence of complex numbers $\left(a_{k}\right)_{k \in \mathbb{Z}}$.

The Fourier transform of the scaling function is given by an infinite product Dau92:

$$
\widehat{\varphi}(x)=\prod_{n=1}^{\infty} m_{0}\left(\frac{x}{2^{n}}\right), \quad(x \in \mathbb{R}) .
$$

The commutant of this wavelet representation can be explicitly computed (see HL00 ): let $\mathcal{F}$ be the Fourier transform. An operator $A$ is in the commutant $\{U, \pi\}^{\prime}$ of the wavelet representation if and only if its Fourier transform $\widehat{A}:=\mathcal{F} A \mathcal{F}^{-1}$ is a multiplication operator by a bounded, dilation invariant function; i.e., $\widehat{A}=M_{f}$, with $f \in L^{\infty}(\mathbb{R}), f(2 x)=f(x)$, for a.e. $x \in \mathbb{R}$. Here

$$
M_{f} \xi=f \xi, \quad\left(\xi \in L^{2}(\mathbb{R})\right) .
$$

Thus, invariant subspaces correspond, through the Fourier transform, to sets which are invariant under dilation by 2 .

The measure $\mu_{\infty}$ on the solenoid $\mathbb{T}_{\infty}$ can also be computed; see Dut06. It is supported on the embedding of $\mathbb{R}$ in the solenoid $\mathbb{T}_{\infty}$. The path measures $P_{x}$ are in this case atomic. 
The direct integral decomposition of the wavelet representation was described in LPT01.

For the low-pass filters that generate non-orthogonal scaling functions, such as the stretched Haar filter $m_{0}(z)=\frac{1}{\sqrt{2}}\left(1+z^{3}\right)$, the wavelet representation can be realized in a finite sum of copies of $L^{2}(\mathbb{R})$. These filters correspond to super-wavelets, and the computation of the commutant of the measure $\mu_{\infty}$ and the direct integral decomposition of the wavelet representation can be found in BDP05, Dut06].

Example 9. Let $r(z)=z^{N}, N \in \mathbb{N}, N \geq 2$, on the unit circle $\mathbb{T}$ and let $m_{0}(z)=1$ for all $z \in \mathbb{T}$. In this case (see [Dut06]) the wavelet representation can be realized on the solenoid $\mathbb{T}_{\infty}$, and the measure $\mu_{\infty}$ is just the Haar measure on the solenoid $\mathbb{T}_{\infty}$, and the operators $U, \pi$ are defined above in the proof of Theorem 3 . For this particular wavelet representation the commutant is trivial, so the representation is irreducible. It is interesting to see that, by Theorem 3 , just any small perturbation of the constant function $m_{0}=1$ will generate a reducible wavelet representation.

Example 10. We turn now to the example in Judy Packer's question: $r(z)=z^{3}$ on $\mathbb{T}$ with the Haar measure, and $m_{0}(z)=\frac{1}{\sqrt{2}}\left(1+z^{2}\right)$. As we explained in the introduction, this low-pass filter generates a wavelet representation involving the middle third Cantor set. See [DJ06] for details. We know that $r(z)=z^{3}$ is an ergodic map, and it is easy to see that the function $m_{0}$ satisfies the hypotheses of Theorem 3. Actually, an application of Jensen's formula to the analytic function $m_{0}^{2}$ shows that

$$
\int_{\mathbb{T}} \log \left|m_{0}\right|^{2} d \mu=-2 \pi \log 2 .
$$

Thus, by Theorem [3, it follows that this wavelet representation is reducible. However, the problem of constructing the operators in the commutant of the wavelet representation remains open for analysis.

\section{REFERENCES}

[BDP05] S. Bildea, D.E. Dutkay, and G. Picioroaga. MRA super-wavelets. New York J. Math. 11 (2005), 1-19 (electronic). MR 2154344 (2006b:42048)

[BFMP09a] L.W. Baggett, V. Furst, K.D. Merrill, and J.A. Packer, Generalized filters, the lowpass condition, and connections to multiresolution analyses, J. Funct. Anal. 257 (2009), no. 9, 2760-2779. MR2559716

[BFMP09b] L.W. Baggett, V. Furst, K.D. Merrill, and J.A. Packer, Classification of generalized multiresolution analyses, J. Funct. Anal. 258 (2010), no. 12, 4210-4228. MR2609543

[BLM09] L.W. Baggett, N.S. Larsen, K.D. Merrill, J.A. Packer, and I. Raeburn, Generalized multiresolution analyses with given multiplicity functions, J. Fourier Anal. Appl. 15 (2009), no. 5, 616-633. MR2563776 (2010j:42071)

[BLP09] L.W. Baggett, N.S. Larsen, J.A. Packer, I. Raeburn, and A. Ramsay, Direct limits, multiresolution analyses, and wavelets, J. Funct. Anal. 258 (2010), no. 8, 2714-2738. MR 2593341 (2011b:42116)

[Dau92] I. Daubechies, "Ten lectures on wavelets", volume 61 of CBMS-NSF Regional Conference Series in Applied Mathematics. Society for Industrial and Applied Mathematics (SIAM), Philadelphia, PA, 1992. MR.1162107(93e:42045)

[DJ06] D.E. Dutkay and P.E.T. Jorgensen. Wavelets on fractals, Rev. Mat. Iberoam. 22 (2006), no. 1, 131-180. MR2268116 (2008h:42071)

[DJ07a] D.E. Dutkay and P.E.T. Jorgensen, Fourier frequencies in affine iterated function systems, J. Funct. Anal. 247 (2007), no. 1, 110-137. MR2319756 (2008f:42007)

[DJ07b] D.E. Dutkay and P.E.T. Jorgensen, Martingales, endomorphisms, and covariant systems of operators in Hilbert space, J. Operator Theory 58 (2007), no. 2, 269-310. MR2358531(2009h:47040) 
[DLS09] D.E. Dutkay, D.R. Larson, and S. Silvestrov, Irreducible wavelet representations and ergodic automorphisms on solenoids, preprint arXiv:0910.0870v2[math.FA], 2009. To appear in Operators and Matrices.

[Dut02] D.E. Dutkay, Harmonic analysis of signed Ruelle transfer operators, J. Math. Anal. Appl. 273 (2002), no. 2, 590-617. MR.1932509 (2003m:42058)

[Dut06] D.E. Dutkay, Low-pass filters and representations of the Baumslag Solitar group, Trans. Amer. Math. Soc. 358 (2006), no. 12, 5271-5291 (electronic). MR2238916 (2007c:42048)

[HL00] D. Han and D.R. Larson, Frames, bases and group representations, Mem. Amer. Math. Soc. 147 (2000), no. 697, x+94 pp. MR.1686653(2001a:47013)

[Jor01] P.E.T. Jorgensen, Ruelle operators: functions which are harmonic with respect to a transfer operator, Mem. Amer. Math. Soc. 152 (2001), no. 720, viii+60 pp. MR.1837681 (2002c:46117)

[LPT01] L.-H. Lim, J.A. Packer, and K.F. Taylor, A direct integral decomposition of the wavelet representation, Proc. Amer. Math. Soc. 129 (2001), no. 10, 3057-3067 (electronic). MR 1840112 (2002c:47146)

Department of Mathematics, University of Central Florida, 4000 Central Florida Boulevard, P.O. Box 161364, Orlando, Florida 32816-1364

E-mail address: ddutkay@mail.ucf.edu

Centre for Mathematical Sciences, Lund University, Box 118, SE-221 00 Lund, SWEDEN

E-mail address: ssilvest@maths.1th.se 